\title{
OPTIMIZATION MODELS FOR A LOT SIZING AND SCHEDULING PROBLEM ON PARALLEL PRODUCTION LINES THAT SHARE SCARCE RESOURCES
}

\author{
Willy Alves de Oliveira Soler ${ }^{1, *}$, Maristela Oliveira Santos ${ }^{2}$ \\ AND SOCORRO RANGEL ${ }^{3}$
}

\begin{abstract}
The purpose of this paper is to propose mathematical models to represent a lot sizing and scheduling problem on multiple production lines that share scarce resources and to investigate the computational performance of the proposed models. The main feature that differentiates this problem from others in the literature is that the decision on which lines to organize should be taken considering the availability of the necessary resources. The optimization criterion is the minimization of the costs incurred in the production process (inventory, backlogging, organization of production lines, and sequence-dependent setup costs). Nine mixed integer optimization models to represent the problem are given and, also, the results of an extensive computational study carried out using a set of instances from the literature. The computational study indicates that an efficient formulation, able to provide high quality solutions for large sized instances, can be obtained from a classical model by making the binary production variables explicit, using the facility location reformulation as well as the single commodity flow constraints to eliminate subsequences. Moreover, from the results, it is also clear that the consideration of scarce resources makes the problem significantly more difficult than the traditional one.
\end{abstract}

Mathematics Subject Classification. 90C11.

Received December 21, 2019. Accepted May 29, 2021.

\section{INTRODUCTION}

The lot sizing problem has been studied since 1913 when it was introduced by Ford Whitman Harris to determine the optimal size of production lots [12]. The scheduling problem consists of determining the order in which the lots are produced in each period of a planning horizon. It was introduced in the 1950s by Johnson's research addressing the flow shop problem (e.g., [10]). These two problems are very important for many companies and they can be addressed using hierarchical approach where the solution obtained from solving the lot sizing problem is used as input data for the scheduling problem. However, as highlighted in [3], this hierarchical approach might result in sub-optimal or even infeasible solutions (i.e., the production levels obtained solving the lot sizing problem cannot be sequenced due to setup times and capacity constraints). Therefore, there is a

Keywords. Lot sizing and scheduling, parallel production lines, scarce resources, mixed integer programming models.

1 Instituto de Matemática, UFMS - Universidade Federal de Mato Grosso do Sul, Campo Grande, MS, Brazil.

2 Instituto de Ciâncias Matemáticas e de Computação, USP - Universidade de São Paulo, São Carlos, SP, Brazil.

3 Departamento de Matemática, UNESP - Universidade Estadual Paulista, São José do Rio Preto 15054-000, SP, Brazil.

*Corresponding author: willy.oliveira@ufms.br 


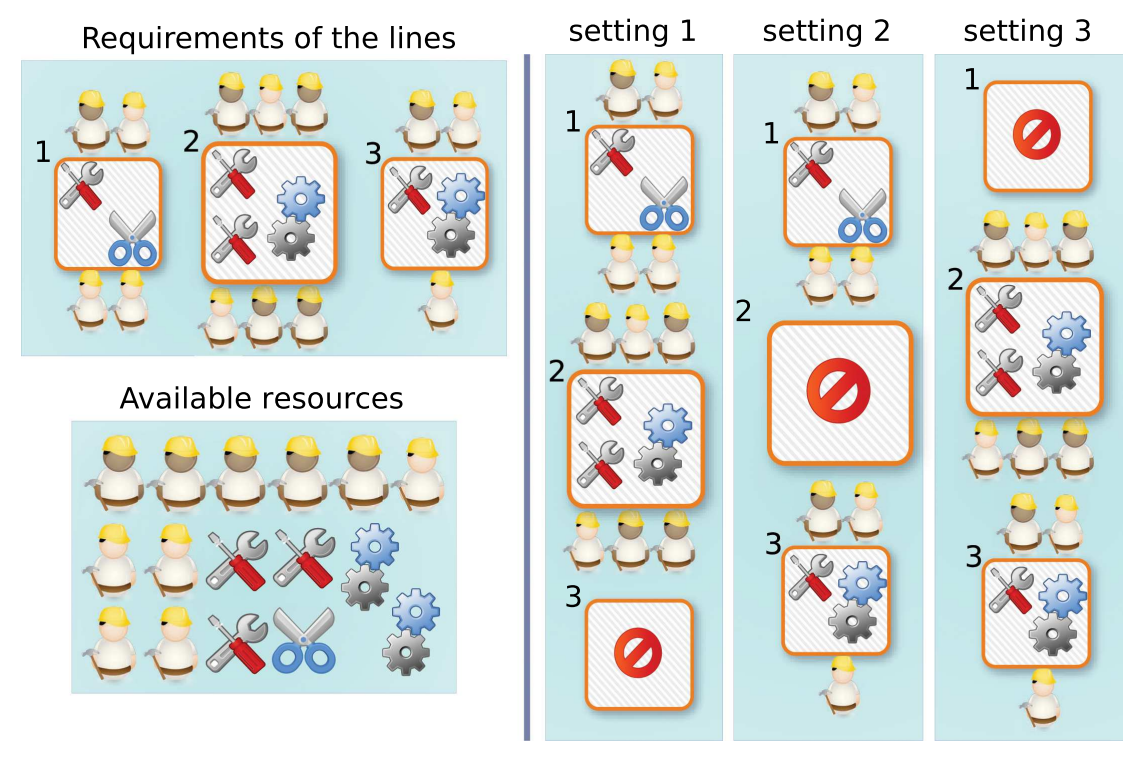

FiguRE 1. Framework of the problem under consideration.

great interest in developing approaches to deal with the simultaneous lot sizing and scheduling problem (LSP). There are various studies in the literature that address the LSP proposing mathematical optimization models and/or specific solutions approaches, for example $[2,4,13,15-17,26]$. Recent reviews of models for the LSP are presented in $[6,13]$.

an extension of the LSP in which multiple production lines share the same scarce resources such as workers, machines, and tools. At the beginning of each period, the resources are assigned to the production lines in order to organize them. Once the lines are organized, they remain so until the end of that period. As there are not enough resources to simultaneously organize all production lines, it is necessary to choose a subset of lines to operate in each period. This aspect of the problem is illustrated in Figure 1: "Requirements of the lines" presents the resources necessary to organize each one of the three production lines considered in this example, while "Available resources" presents the production resources available in the factory. Due to the scarcity of the resources, it is not possible to simultaneously organize all three production lines. Therefore, in each period, the managers need to choose one of the three production layouts presented in "setting 1", "setting 2" and "setting 3 " to produce the items. Moreover, we suppose that the lines are specialized, in other words, for each product, only a subset of lines is able to produce it. Thus, the lines chosen to be organized in any given period impacts the set of products that can be produced in that period. The problem is also characterized by the existence of backlogging (penalized in the objective function) and sequence dependent setup times and costs.

This problem was introduced in [24] motivated by the production process adopted by some Brazilian food companies. To handle the problem, mixed integer mathematical optimization (MIP) models and a MIP-based heuristic were proposed in [24]. The heuristic was able to provide good solutions for the problem, outperforming the branch-and-cut algorithm of a commercial solver and others MIP-based heuristics from the literature within a limited running time of several hours. We note that, in [24], only two models were proposed with a lack of results about their computational efficiency considering alternative formulations. Besides that, the two models consider specific characteristics of the food industry, such as perishable products. Our paper considers the sharing of scarce resources across multiple production lines in a general framework that can be easily extended to deal with problems arising in a range of different production environments. Moreover, among the different formulations proposed in this paper, there is one that significantly outperforms the models used in [24]. 
Summarizing, the problem considered in this paper consists in simultaneously deciding: i) which production lines to organize in each production period; ii) the sequence in which the items are produced on each line; and iii) the size of the production lots; ensuring that the capacity constraints of the production resources necessary to organize the lines and the production capacity constraints of the lines to be used are respected. The optimization criterion considered is the minimization of the total costs incurred in the production process. The problem is denoted as Lot sizing and Scheduling Problem on Parallel Lines Sharing Scarce Resources (LSP-PLSR). Besides the application of this problem to the production planning in a meat company (as described in [24]), the sharing of scarce resources by multiple production lines also occur in other industrial contexts such as in the automotive industry [14], semiconductor and soft drinks industries [4], as well as the block erection scheduling problem in a shipyard [1].

In this paper we propose nine mathematical optimization models for the LSP-PLSR. We begin by extending the GLSP (General Lot Sizing and scheduling Problem [9]) and the CLSD (Capacitated Lot sizing with Sequence Dependent setup costs problem [15]), two of the most used models in the literature for the LSP, to consider the LSP-PLSR. The other seven models are obtained from reformulations of these models (two reformulations for the GLSP model and five for the CLSD model) in order to study the computational impact of some modeling strategies regarding three aspects:

- explicit use of binary variables to model the setup states of the lines (for short: binary production variables). Originally, in the GLSP model these variables are explicit, while in the CLSD model they are implicit. Therefore, we have developed reformulations making these variables implicit (explicit) in the GLSP (CLSD) model;

- sequencing modelling strategy. In the CLSD model, constraints are used to eliminate solutions presenting disconnected sub-sequences and most papers in the literature use the traditional MTZ constraints for this purpose. In this paper, we also investigate the use of single commodity flow (SCF) constraints proposed in [11]. Moreover, through the GLSP model, we can study the micro period strategy to model the sequencing decisions;

- facility location (FL) reformulation of the lot sizing decisions. This strategy was proposed in [8] to model the production variables associated with the lot sizing decisions. For the LSP-PLSR, the performance of this reformulation may depends of other aspects. Therefore, we study the FL reformulation for some of the models obtained.

Besides proposing nine mathematical optimization models combining modeling strategies that has not yet been considered simultaneously in the literature for the LSP-PLSR, our contributions also comprise an extensive computational study that includes performance and sensitivity analysis. In the performance analysis, we identify the most promising formulation and study the computational impact of each adopted modeling strategy, while in the sensitivity analysis, we study the computational impact of the scarcity rate of the production resources.

The remainder of the paper is organized as follows. Section 2 presents the related researches with emphasis on the mathematical optimization models that serve as the basis for the nine proposed MIP models presented in Section 3. In Section 4 we present the computational study framework and the associated results. Finally, Section 5 concludes the paper by highlighting our main contributions and indicating potential directions for future researches.

\section{LiteratuRE REVIEW}

The simultaneous lot sizing and scheduling problem (LSP) consists of determining the sizes and the sequence of production lots in order to minimize the production costs and meet customer demands. The LSP can be represented by different mixed integer programming models. Some of the most general-purpose models for the LSP were proposed in $[5,9,15,20]$.

In [9], the general lot sizing and scheduling (GLSP) model was introduced considering that multiple items (with demands known for each period of a finite planning horizon) need to be produced on a single machine. The 
GLSP model also considers sequence-dependent setup costs and production capacity constraints. The main idea of the GLSP model is to split each period into several smaller periods (micro periods) with varying sizes and such that only one item can be produced in each micro period. By defining which items are produced in each micro period, the production lot sequence is then automatically determined. The GLSP model was reformulated in [25] by using constraints from models for the network flow problem to model the sequencing decisions. In [5], the GLSP model was also reformulated by suppressing the setup state variables (CC model).

The GLSP model was extended in [21] to consider multiple production lines (GLSPPL model). In the GLSPPL model, each production line is considered as an automatic flow between production processes and workstations, so that each production line can be considered as a single machine. In the original GLSPPL model, all products can be produced on any production line and the lines are always prepared for production.

Instead of splitting the periods into micro periods, the capacitated lot sizing with sequence dependent setup costs (CLSD) model proposed by [15] uses the structure of the mathematical models for the travelling salesman problem (TSP) to obtain the sequencing of the lots. More specifically, they used the MTZ (Miller, Tucker and Zemlim, e.g., [19]) subtour elimination constraints. Recently, in [13], the GLSP, CC, and CLSD models were studied with respect to the computational performance when they were solved by a Branch-and-Cut algorithm from a commercial solver. The authors also analyzed the reformulation based on the facility location problem, proposed in [8] for these models. Additionally, for the CLSD model, different constraints for sub-sequences elimination were considered instead of the traditional MTZ constraints (used in [15]). The results presented in [13] indicate that for the single machine LSP:

- the reformulation proposed in [25] performs better than the original GLSP model;

- the CC model performs better than the reformulation proposed in [25];

- the facility location reformulation improves the performance of the CC and CLSD models, but it is not useful for the GLSP model;

- the CLSD model performs better using the single commodity flow (SCF) constraints than using the MTZ constraints;

- the performance of the CC and CLSD models are very similar. More specifically, the CC model obtained better solutions than CLSD model (using the MTZ constraints), while the CLSD model obtained better solutions than the CC model when the SCF constraints were used instead of the MTZ constraints.

To the best of our knowledge the computational behavior of these different strategies used to obtain the sequence of lots has not been compared in the case of the LSP with parallel production lines.

In the literature, there are various strategies to mathematically represent problems based on observations made in real-world production environments. Many of them extend some of the basic models already mentioned and add variables and constraints to deal with the additional characteristics from the specific production environment being considered. For example, in [18], the CLSD model was extended to address an LSP in a yogurt factory which operates parallel machines and groups the items into product families. In [26], the CLSD model was adapted to deal with an LSP on parallel machines where each item can be produced on all machines but, for each item, there is a preferred machine for production and a bonus is computed in the objective function when an item is produced on its preferred machine. Recently, in [2] the GLSP was extended to consider companies that produce perishable food products on multiple parallel production lines.

Among the papers addressing problems similar to the LSP-PLSR, we highlight $[1,4]$. In [4] the GLSP and the CLSD models were extended to consider an industrial environment where parallel production lines need to be equipped with secondary resources (tools) for processing and due to the scarcity of these secondary resources, their use was synchronized along the production lines. This problem differs from the LSP-PLSR because all production lines can simultaneously operate, but for each line configuration, only some products can be produced. Besides that, in [4] only two out of the nine strategies studied here are considered. In [1], a scheduling problem was addressed in which several production lines share scarce secondary production resources, such as, labor, tools, pallets, and industrial robots. The aim is to minimize the makespan and the lot sizing 
TABLE 1. Parameters for the LSP-LPSR.

\begin{tabular}{ll}
\hline \hline$T, L$ & Number of periods (indexed by $t$ and $t^{\prime}$ ), and of production lines (indexed by $l$ ) \\
$S_{l t}$ & Set of micro-periods in period $t$ and on line $l$ (only for the \\
& GLSP model and its reformulations). The micro-periods are indexed by $s$ \\
$J, K$ & Number of products (indexed by $i, j$ ), and of resources (indexed by $k$ ) \\
$d_{j t}, C_{l t}$ & Demand of product $j$, and production capacity (in minutes) of line $l$ in period $t$ \\
$a_{l j}, m_{l j}$ & Production time per unit and the minimum lot size of product $j$ on line $l$ \\
$h_{j}, b_{j}$ & Inventory and backlogging costs per unit of product $j$ \\
$s c_{l i j}, s t_{l i j}$ & Setup cost and time for changeover from product $i$ to $j$ on line $l$ \\
$r_{k l}$ & Amount of resource $k$ necessary to organize production line $l$ \\
$R_{k t}$ & Capacity of resource $k$ in period $t$ \\
$a c_{l}$ & Cost of organizing line $l$ \\
$\gamma_{l t}$ & Maximum number of products that can be produced on line $l$ in period $t$ \\
$P_{l}$ & Set of products that can be produced on line $l$ \\
\hline
\end{tabular}

aspect is not addressed. Moreover, only one model was considered without presenting a discussion about the efficiency of the used modeling strategy and heuristic approaches were developed to find feasible solutions.

The LSP-PLSR was introduced in [24]. The authors have proposed an extension of the CLSD model and a reformulation exploring only one modeling aspect (explicit use of binary variables to model the setup states of the lines) the production binary variables) with a lack of results about the efficiency of those models. Moreover, a very successful relax-and-fix heuristic was developed able to deliver high quality feasible and dual solutions. In this paper, we study and compare different modeling strategies to represent the LSP-PLSR with the aim of obtaining models better than the formulations proposed in [24]. In this case, we note that as the relax-and-fix heuristic proposed in [24] depends of the mathematical formulation of the problem, it might be improved by using a better model. Moreover, the modeling insights obtained in this study can be used to develop stronger models to deal with similar problems such as the problems addressed in $[1,4]$.

In Section 3, the mathematical models mentioned above are extended to represent the LSP-PLSR. Novel model proposals are presented based on variations of these traditional models exploring modeling aspects related to: i) expliciting the binary production variables; ii) sequence decisions; and iii) the facility location reformulation of the lot sizing decisions.

\section{MODELS FOR THE LSP-PLSR}

In this section we present nine models to represent the LSP-PLSR. Two of them are extensions of the general models GLSP and CLSD to consider the particularities of the LSP-PLSR (Sect. 3.1). These two models were specifically chosen because they have been extensively and successfully used in the literature to deal with LSPs and, through these two models, we can study different strategies to model the sequence decisions in the LSPPLSR. Another two models are based on reformations of the GLSP as proposed in [5] and in [8] (Sect. 3.3). The other five models are obtained by reformulations of the CLSD model inspired in the proposals given in $[8,11,13,23]$ (Sect. 3.6). The main characteristics of the proposed models and a preliminary analysis with respect to their total number of continuous/binary variables are given in Section 3.8. The constraints (and objective functions) referring to the GLSP based models are labeled by (G.) while the constraints referring to the CLSD based models are labeled by (C.). The constraints used in both models are labeled by (B.) and the auxiliary constraints that are not explicitly used in the models are labeled by (A.).

\subsection{General models: GLSP and CLSD}

In this section, we extend the general models GLSP [21] and CLSD [15] to consider the LSP-PLSR. Table 1 presents the parameters that are used to define the models, while the decision variables are shown in Table 2. 
TABLE 2. Decision variables for the GLSP and CLSD models.

\begin{tabular}{|c|c|c|}
\hline \multicolumn{2}{|c|}{ Common variables } & Description \\
\hline \multirow{3}{*}{\multicolumn{2}{|c|}{$\begin{array}{c}I_{j t} \\
B_{j t} \\
\delta_{l t}\end{array}$}} & Inventory level of product $j$ at the end of period $t$ \\
\hline & & Backlogging level of product $j$ at the end of period $t$ \\
\hline & & 1 if line $l$ is organized in period $t$ and 0 otherwise \\
\hline \multicolumn{2}{|c|}{ Specific variables } & Description \\
\hline GLSP & CLSD & \\
\hline$x_{l j s}$ & $x_{l j t}$ & Amount of item $j$ produced on line $l$ in micro period $s /$ period $t$ \\
\hline$z_{\text {lijs }}$ & $z_{l i j t}$ & $\begin{array}{l}1 \text { if there is change of production from product } i \text { to product } j \\
\text { on line } l \text { in micro period } s / \text { period } t \text { and } 0 \text { otherwise }\end{array}$ \\
\hline$w_{l j s}$ & $w_{l j t}$ & $\begin{array}{l}1 \text { if line } l \text { is setup for product } j \text { in micro period } s / \text { period } t \\
\text { and } 0 \text { otherwise }\end{array}$ \\
\hline- & $y_{l j t}$ & $\begin{array}{l}1 \text { if item } j \text { is the first item produced on line } l \text { in period } t \\
\text { and } 0 \text { otherwise }\end{array}$ \\
\hline- & $V_{l j t}$ & Order of production of item $j$ on line $l$ in period $t$ \\
\hline
\end{tabular}

- Objective function. The objective function consists of minimizing the total costs incurred in the production process. More specifically, we consider the inventory holding, backlogging and setup costs and the costs of organizing the production lines. Therefore, in the GLSP model, the objective function is given by (3.1), while, in the CLSD model, it is given by (3.2).

$$
\begin{aligned}
& \operatorname{Min} \sum_{j} \sum_{t}\left(h_{j} I_{j t}+b_{j} B_{j t}\right)+\sum_{l} \sum_{i \in P_{l}} \sum_{j \in P_{l}} \sum_{s \in S_{l t}} s c_{l i j} z_{l i j s}+\sum_{l} \sum_{t} a c_{l} \delta_{l t} \\
& \operatorname{Min} \sum_{j} \sum_{t}\left(h_{j} I_{j t}+b_{j} B_{j t}\right)+\sum_{l} \sum_{i \in P_{l}} \sum_{j \in P_{l}} \sum_{t} s c_{l i j} z_{l i j t}+\sum_{l} \sum_{t} a c_{l} \delta_{l t} .
\end{aligned}
$$

- Inventory balance constraints. The inventory balance constraints for the GLSP and CLSD models are given by (3.3) and (3.4), respectively.

$$
\begin{array}{ll}
I_{j t}-B_{j t}=I_{j, t-1}-B_{j, t-1}+\sum_{l, s \in S_{l t}} x_{l j s}-d_{j t}, & \forall j, t \\
I_{j t}-B_{j t}=I_{j, t-1}-B_{j, t-1}+\sum_{l} x_{l j t}-d_{j t} . & \forall j, t
\end{array}
$$

Besides that, we need to ensure that the customer demands are fully met until the end of the planning horizon. Therefore, we include the constraints (3.5) in both models.

$$
B_{j T}=0 . \quad \forall j
$$

- Capacity constraints. We consider that each organized production line has a limited production capacity (time) in each period. For each period $t$ and production line $l$, constraints $(3.6) /(3.7)$ ensure that the sum of the time consumed with the production of items and with the setup procedures does not exceed the available production capacity $C_{l t}$.

$$
\begin{array}{ll}
\sum_{j \in P_{l}, s \in S_{l t}} a_{l j} x_{l j s}+\sum_{i, j \in P_{l}, s \in S_{l t}} s t_{l i j} z_{l i j s} \leq C_{l t}, & \forall l, t \\
\sum_{j \in P_{l}} a_{l j} x_{l j t}+\sum_{i, j \in P_{l}} s t_{l i j} z_{l i j t} \leq C_{l t} . & \forall l, t
\end{array}
$$


- Setup state and minimum lot sizes constraints. Constraints (3.8)/(3.9) ensure that each product can only be produced if the respective production line is setup for that item. Moreover, these constraints introduce the minimum lot sizes $m_{j}$, i.e., if line $l$ is set up for item $j$ at least $m_{j}$ units of item $j$ need to be produced on that line. Note that in the LSP-PLSR addressed here, setup carry-over is not allowed because the resources can be removed from the lines at the end of the periods, and when this happens, the setup state of the lines are dropped. Hence, for the first micro period of each period $t$ and line $l\left(f_{l t}\right)$ constraints (3.10) are used instead of (3.8).

$$
\begin{aligned}
& m_{j}\left(w_{l j s}-w_{l j, s-1}\right) \leq x_{l j s} \leq \frac{C_{l t}}{a_{l j}} w_{l j s}, \forall l, t, j \in P_{l}, s \in S_{l t} \backslash\left\{f_{l t}\right\} \\
& m_{j}\left(y_{l j t}+\sum_{i \in P_{l} \backslash\{j\}} z_{l i j t}\right) \leq x_{l j t} \leq \frac{C_{l t}}{a_{l j}}\left(y_{l j t}+\sum_{i \in P_{l} \backslash\{j\}} z_{l i j t}\right), \forall l, j, t \\
& m_{j} w_{l j s} \leq x_{l j s} \leq \frac{C_{l t}}{a_{l j}} w_{l j s}, \forall l, t, j \in P_{l}, s=f_{l t}
\end{aligned}
$$

- Sequencing constraints. In the GLSP model, we include constraints (3.11) to capture the changes of production on each line for each period. More specifically, constraints (3.11) ensure that if production line $l$ is set up for item $j$ in a micro period $s$ (i.e., $w_{l j s}=1$ ) and it was set up for item $i$ (with $i \neq j$ ) in the previous micro period $s-1\left(w_{l i, s-1}=1\right)$, then there is a change of production from item $i$ to $j$ on line $l$ for micro period $s$ (i.e., $\left.z_{l i j s}=1\right)$.

$$
z_{l i j s} \geq w_{l i, s-1}+w_{l j s}-1, \forall l, t, i, j \in P_{l}, s \in S_{l t} \backslash\left\{f_{l t}\right\} .
$$

Since setup carry-over is not allowed, constraints (3.11) are not used in the first micro period of each period and line. Moreover, we consider that the setup procedures to produce the first product in a given period and line can be made together with the procedures to organize the lines without increase in time/cost. Hence, the setup time and cost to produce the first item in each period and line are not considered.

In the CLSD model, the sequencing decisions are modeled using constraints (3.12), (3.13), and (3.14). More specifically, constraints (3.12) impose that if a production line is organized, only one product can be the first one to be produced on that line, while constraints (3.13) are the flow balance for sequencing of lots. Constraints (3.14) are the MTZ constraints, proposed in [22] and originally used by [15] in the CLSD model, to eliminate solutions presenting disconnected sub-sequences. The auxiliary variables $V_{l j t}$ can be interpreted as an indication of the position in which product $j$ is produced on line $l$ and period $t$. Constraints (3.14) then ensure that if there is change of production from product $i$ to product $j$, then the position of product $j$ is greater than the position of product $i$ in the production sequence.

$$
\begin{array}{ll}
\sum_{j \in P_{l}} y_{l j t} \leq \delta_{l t}, & \forall l, t \\
y_{l j t}+\sum_{i \in P_{l} \backslash\{j\}} z_{l i j t} \geq \sum_{i \in P_{l}} z_{l j i t}, & \forall l, j \in P_{l}, t \\
V_{l j t} \geq V_{l i t}+1-\gamma_{l t}\left(1-z_{l i j t}\right) . & \forall l, t, i, j, i \neq j
\end{array}
$$

- Constraints to ensure that the products are produced only on organized production lines. In the GLSP model, constraints (3.15) ensure that the products can only be produced on organized lines and that, in each micro period, the organized lines are set up for exactly one item. Indeed, since $w_{l j s} \in\{0,1\}, \forall l, j, s$ and $\delta_{l t} \in\{0,1\}, \forall l, t$, given line $l$ and period $t$, by constraints (3.15) $w_{l j s}=0, \forall j, s \in S_{l t}$ if and only if $\delta_{l t}=0$, i.e., there is no production on line $l$ when this line is not organized. On the other hand, constraints (3.15) also ensure that if $\delta_{l t}=1$ then for each $s \in S_{l t}$ there exists a product $j$ such that $w_{l j s}=1$, i.e., the organized 
production lines are always set up for one product.

$$
\sum_{j \in P_{l}} w_{l j s}=\delta_{l t} . \quad \forall l, t, s \in S_{l t}
$$

Let $S_{l t}=\left\{f_{l t}, \ldots, u_{l t}\right\}$ the set of micro periods in period $t$ for line $l$. The assumption that at most $\gamma_{l t}$ different products can be produced on each line in a given period is stated in the GLSP model ensuring that $u_{l t}-f_{l t}=\gamma_{l t}-1, \forall l, t$ in the input data.

As for the CLSD model, constraints (3.16) ensure that at most $\gamma_{l t}-1$ changes of products can be made on each organized line and period while there are no changes of products on lines not being used. Therefore, constraints (3.16) together with constraints (3.14) ensure that a maximum of $\gamma_{l t}$ products can be produced on each organized line and period and that there is no production on lines not being used.

$$
\sum_{i, j \in P_{l}} z_{l i j t} \leq\left(\gamma_{l t}-1\right) \delta_{l t} . \quad \forall l, t
$$

- Capacity constraints of the production resources. In this problem, we need to ensure that the amount of each resource $k$ used in each period to organize the production lines does not exceed the availability of that resource. Therefore, we include constraints (3.17) in the both models.

$$
\sum_{l} r_{k l} \delta_{l t} \leq R_{k} . \quad \forall k, t
$$

- Domain of the decision variables. Constraints (3.18)-(3.20) and (3.21)-(3.23) define the domain of the specific variables used in the GLSP and CLSD models, respectively.

$$
\begin{array}{ll}
x_{l j s} \geq 0, & \forall l, j, s \\
w_{l j s} \in\{0,1\}, & \forall l, j, s \\
0 \leq z_{l i j s} \leq 1, & \forall l, i, j, s \\
x_{l j t} \geq 0, & \forall l, j, t \\
y_{l j t} \in\{0,1\}, & \forall l, j, t \\
z_{l i j t} \in\{0,1\} . & \forall l, i, j, t
\end{array}
$$

Note that, in constraints (3.20) the variables $z_{l i j s}$ are defined as continuous even though they can only assume the values 0 or 1 . As discussed in [9] this is possible due to constraints (3.11) and (3.19) and the fact that the variables $z_{l i j s}$ are penalized in the objective function.

Finally, the domain of the common variables are given by constraints (3.24) and (3.25).

$$
\begin{aligned}
& I_{j t} \geq 0, B_{j t} \geq 0, \quad \forall j, t \\
& \delta_{l t} \in\{0,1\} . \quad \forall l, t
\end{aligned}
$$

- Optional constraints for the GLSP model. In [9], the authors have observed that the GLSP model admits undesirable redundancy. If a set of consecutive micro periods within a given period is assigned to the same product, then the production quantity of this lot could be distributed among these micro periods without change in the objective function value and the production sequence. Therefore, to avoid this type of redundancy we have extended the constraints proposed in [9] as described in (3.26) and (3.27).

$$
\begin{aligned}
& \sum_{i \in P_{l}} \sum_{j \in P_{l} \backslash\{i\}} z_{l i j s-1} \geq \sum_{i \in P_{l}} \sum_{j \in P_{l} \backslash\{i\}} z_{l i j s}, \quad \forall l, t, s=f_{l t}+2, \ldots, u_{l t} \\
& x_{l j s} \leq \frac{C_{l t}}{a_{l j}}\left(2-\sum_{i \in P_{l}} z_{l i j s-1}-z_{l j j s}\right) . \quad
\end{aligned}
$$




\subsection{Summary of the models GLSP and CLSD for the LSP-PLSR}

In this section we have extended two general models from the literature (based on [15, 21]) to represent the LSP-PLSR. For simplicity, in this paper we denote the models obtained here using the same initials as those for the original models from the literature, i.e., GLSP and CLSD. The complete models are: GLSP: $\quad$ Min (3.1) s.t.: $\quad(3.3)-(3.27)$, and (3.5)-(3.25);

CLSD: $\quad$ Min (3.2) s.t.: $\quad(3.4)-(3.23)$, and (3.5)-(3.25).

\subsection{Reformulations of the GLSP model}

Two reformulations for the GLSP model are given in this section. The first one, presented in Section 3.3.1, is an extension of the CC model proposed in [5] and it is denoted in this paper by GLSP ${ }^{C C}$. The GLSP ${ }^{C C}$ model is built by eliminating the binary variables that define the setup states of the lines $\left(w_{l j s}\right)$. The second one, presented in Section 3.4.1, is a reformulation of the GLSP ${ }^{C C}$ model using the structure of the facility location problem as proposed in [8] and it is denoted by FL-GLSPCC

\subsubsection{The GLSPCC model}

The GLSPCC model is an extension of the CC model proposed in [5]. This model is characterized by suppressing the binary setup state variables $w_{l j s}$. Note that, item $j$ is produced on line $l$ in period $t$ if, and only if a change of product is performed from any item $i$ to item $j$, i.e.,

$$
w_{l j s}=\sum_{i \in P_{l}} z_{l i j s}, \quad \forall l, j \in P_{l}, s .
$$

Therefore, the GLSP ${ }^{C C}$ model is obtained from the GLSP model using the equation (3.28) to suppress the variables $w_{l j s}$. More specifically:

- The setup state and maximum lot sizes constraints (3.8) and (3.10) are replaced by (3.29) and (3.30). As we have observed in Section 3.1, setup carry-over is not allowed. Therefore, in the first micro period of each period and line, the minimum lot sizes need to be respected independent of the product that was produced in the previous micro period (last micro period of the previous period). That is why we have addressed the minimum lot sizes of the first micro periods in constraints (3.30) instead of (3.29).

$$
\begin{array}{ll}
m_{l j} \sum_{i \in P_{l}, i \neq j} z_{l i j s} \leq x_{l j s} \leq \frac{C_{l t}}{a_{l j}} \sum_{i \in P_{l}} z_{l i j s}, & \forall l, j \in P_{l}, t, s \in S_{l t} \backslash\left\{f_{l t}\right\} \\
m_{l j} \sum_{i} z_{l i j s} \leq x_{l j s} \leq \frac{C_{l t}}{a_{l j}} \sum_{i \in P_{l}} z_{l i j s} . & \forall l, j \in P_{l}, s=f_{l t}
\end{array}
$$

- The sequencing constraints (3.11) are replaced by constraints (3.31).

$$
\sum_{i} z_{l i j, s-1}=\sum_{i} z_{l j i s}, \forall l, t, i \in P_{l}, j \in P_{l}, s \in S_{l t} \backslash\left\{f_{l t}\right\} .
$$

Constraints (3.31) ensure that a changeover from item $j$ to another item can only occur in micro period $s$ if a changeover from some other item to item $j$ has occurred in the previous micro period $s-1$.

- The constraints (3.15) are replaced by constraints (3.32) in order to ensure that the items can only be produced on organized production lines. These constraints also ensure that for the lines being used, only one changeover can occur in each micro period.

$$
\sum_{i \in P_{l}} \sum_{j \in P_{l}} z_{l i j s}=\delta_{l t} . \quad \forall l, t, s \in S_{l t}
$$

- As the binary variables $w_{l j s}$ are suppressed in the GLSPCC model, we need to ensure that the variables $z_{l i j s}$ assume only the values 0 or 1 . Therefore, we replace constraints (3.20) by constraints (3.33).

$$
z_{l i j s} \in\{0,1\} . \quad \forall l, i, j, s
$$




\title{
3.4. Summary of the model GLSPCC for the LSP-PLSR
}

\author{
GLSPCC$^{\mathrm{CC}} \quad \operatorname{Min}(3.1)$ s.t.: (3.3), (3.6), and (3.29)-(3.32), (3.18),
}

$(3.33),(3.26),(3.27)$ and $(3.5)-(3.25)$.

\subsubsection{The FL-GLSPCC model}

In [8], the lot sizing problem was modeled using the structure of the facility location problem. The authors observed that the linear relaxation of this model is very effective in generating good dual bounds. The computational study reported in [13] showed that the facility location reformulation also provided good results for the single machine lot sizing and scheduling problem. In this paper, we investigate the performance of the facility location reformulation of the GLSP ${ }^{\mathrm{CC}}$ model to address the LSP-PLSR (model FL-GLSPCC).

To obtain the new model, the variables $x_{l j s}$ are replaced by $x_{l j s t}$ to indicate the amount of item $j$ produced on line $l$ during micro period $s$ to met the demand in period $t$. Using this approach, there is no need to include the variables that explicitly account for the inventories $\left(I_{j t}\right)$ and the backloggings $\left(B_{j t}\right)$ of the items. These values are given by the non-negative difference between the sum of the production of item $j$ in the previous periods and the sum of the demands previously met, as stated in equations (3.34) and (3.35) for the inventory and for the backlogging levels of product $j$ in each period $t$ respectively.

$$
I_{j t}=\max \left\{\sum_{l} \sum_{s \in \cup_{t^{\prime} \leq t} S_{l t^{\prime}}} x_{l j s t}-\sum_{t^{\prime} \leq t} d_{j t^{\prime}}, 0\right\}, \quad \forall j, t
$$

and

$$
B_{j t}=\max \left\{\sum_{t^{\prime} \leq t} d_{j t^{\prime}}-\sum_{l} \sum_{s \in \cup_{t^{\prime} \leq t} S_{l t^{\prime}}} x_{l j s t}, 0\right\} . \forall j, t
$$

To compute the inventory or backlogging costs $\left(h c_{l j s t}\right)$ incurred in producing a unit of item $j$ on line $l$ during micro period $s$ to meet the demand in period $t$, let $\lambda_{l s}$ be the period that contains the micro period $s$ of line $l$, i.e., $\lambda_{l s}=t$ if and only if $s \in S_{l t}$. Then for $\lambda_{l s} \leq t, h c_{l j s t}=h_{j}\left(t-\lambda_{l s}\right)$ is the inventory cost, and for $\lambda_{l s}>t$, $h c_{l j s t}=b_{j}\left(\lambda_{l s}-t\right)$ is the backlogging cost. The FL-GLSPCC model is obtained from the GLSPCC model by modifying the objective function (3.1) and some constraints as follows.

- The objective function (3.1) is rewritten in terms of the new set of variables $x_{l j s t}$ and is replaced by (3.36). Note that the inventory and backlogging costs are computed in the first sum of (3.36).

$$
\operatorname{Min} \sum_{l, j, s, t} h c_{l j s t} x_{l j s t}+\sum_{l, i, j, s} s c_{l i j} z_{l i j s}+\sum_{l, t} a c_{l} \delta_{l t} .
$$

- As in the FL-GLSPCC we do not consider the variables $I_{j t}$ and $B_{j t}$, the inventory balance constraints (3.3) need to be adapted according to (3.37).

$$
\sum_{l} \sum_{s \in \cup_{t} S_{l t}} x_{l j s t}=d_{j t} . \quad \forall j, t
$$

- The capacity constraints (3.6) also need to be adapted due to the redefinition of the production variables $x_{\text {ljst }}$.

$$
\sum_{j \in P_{l}, s \in S_{l t}} a_{l j} x_{l j s t^{\prime}}+\sum_{i, j \in P_{l}, s \in S_{l t}} s t_{l i j} z_{l i j s} \leq C_{l t} . \quad \forall l, t
$$


- The setup state and maximum lot sizes constraints (3.29) and (3.30) are replaced by constraints (3.39) and (3.40) due to the redefinition of the production variables.

$$
\begin{array}{ll}
m_{l j} \sum_{i \in P_{l}, i \neq j} z_{l i j s} \leq \sum_{t^{\prime}} x_{l j s t^{\prime}} \leq \frac{C_{l t}}{a_{l j}} \sum_{i \in P_{l}} z_{l i j s}, & \forall l, j, t, s \in S_{l t} \backslash\left\{f_{l t}\right\} \\
m_{l j} \sum_{i} z_{l i j s} \leq \sum_{t^{\prime}} x_{l j s t^{\prime}} \leq \frac{C_{l t}}{a_{l j}} \sum_{i \in P_{l}} z_{l i j s} . & \forall l, j \in P_{l}, s=f_{l t}
\end{array}
$$

- The domain constraints (3.18) are replaced by (3.41).

$$
x_{l j s t} \geq 0 . \quad \forall l, j, s, t
$$

- The optional constraints (3.27) are slightly modified and replaced by constraints (3.42).

$$
\sum_{t^{\prime}} x_{l j s t^{\prime}} \leq \frac{C_{l t}}{a_{l j}}\left(2-\sum_{i \in P_{l}} z_{l i j s-1}-z_{l j j s}\right) . \quad \forall l, j, t, s \in S_{l t} \backslash\left\{f_{l t}\right\}, t^{\prime}
$$

\subsection{Summary of the model FL-GLSP ${ }^{C C}$ for the LSP-PLSR

$$
\begin{aligned}
\text { FL-GLSPCC: } & \text { Min (3.36) s.t.: } \\
& (3.37),(3.38),(3.39),(3.40),(3.31), \\
& (3.32),(3.41),(3.33),(3.26), \\
& (3.42),(3.17),(3.25)
\end{aligned}
$$

\subsection{Reformulations for the CLSD model}

The five reformulations for the CLSD model presented in this section are based on three main ideas: i) including binary variables to indicate explicitly if a line is (or is not) set up to produce an item in each period as suggested in [23] (Sect. 3.6.1), ii) using the SCF constraints instead of the MTZ constraints to eliminate subsequences as suggested in [13] (Sect. 3.7.1), and iii) using the facility location reformulation based on [8] (Sect. 3.7.2).

\subsubsection{The $C L S D^{w}$ model}

In the CLSD model we do not use explicit binary variables to indicate the set up state of the lines. This information is implicitly considered through the variables $\left(y_{l i t}, z_{l i j t}\right)$. However, as seen in [23], the explicit utilization of set up variables can help the Branch-and-cut algorithm to explore the feasible set of the problem more efficiently as is explained next.

Consider that the set up states of the lines in each period are captured using the variables $w_{l j t}$ as defined in (3.43).

$$
w_{l j t}=y_{l j t}+\sum_{i \in P_{l}} z_{l i j t} . \quad \forall l, j, t .
$$

Suppose that, in a node of the search tree of a Branch-and-cut algorithm we have fixed $w_{l j t}=0$ for some $l, j, t$. Then, by (3.43) together with (3.22) and (3.23) we have that $y_{l j t}=0$ and $z_{l i j t}=0, \forall i$. Besides that, $y_{l j t}=0$ and $z_{l i j t}=0, \forall i$ together with (3.13) imply that $z_{l j i t}=0 \forall i$. Therefore, fixing the value of the binary variable $w_{l j t}$ to zero implies that the value of the other $1+2\left|P_{l}\right|$ binary variables are also zero. The Branch-and-cut algorithms from some commercial solvers (like Cplex) can identify this structure of the model and might use it to branch on the binary variables $w_{l j t}$ before the others, so reducing the running time and average GAPs (e.g., [23]).

The $\mathrm{CLSD}^{w}$ model can be obtained from the CLSD model by:

- introducing the constraints (3.43) and constraints (3.44) in order to define the domain of the new decision variables;

$$
w_{l j t} \in\{0,1\}, \quad \forall j, t
$$


- replacing the setup state and minimum lot sizes constraints (3.9) by (3.45);

$$
m_{j} w_{l j t} \leq x_{l j t} \leq \frac{C_{l t}}{a_{j}} w_{l j t}, \quad \forall l, j, t
$$

- replacing the constraints to ensure that the products are produced only on organized production lines (3.16) by $(3.46)$.

$$
\sum_{j \in P_{l}} w_{l j t} \leq \gamma_{l t} \delta_{l t}, \quad \forall l, t
$$

\subsection{Summary of the model CLSD ${ }^{w}$ for the LSP-PLSR}

$$
\begin{aligned}
\mathrm{CLSD}^{w}: \quad \text { Min (3.2) s.t.: } & (3.4),(3.7),(3.45),(3.12),(3.13),(3.14), \\
& (3.46),(3.21)-(3.44), \text { and }(3.5)-(3.25)
\end{aligned}
$$

\subsubsection{The $C L S D_{\mathrm{SCF}}$ and $C L S D_{\mathrm{SCF}}^{w}$ models}

The CLSD model was introduced in [15] using the MTZ constraints in order to eliminate subsequences in the production sequence. However, in the literature, there are a broad range of other constraints that also do the same job, among which we highlight the single commodity flow (SCF) constraints proposed in [11]. As observed in [13], the SCF constraints can improve the computational performance of the single machine CLSD model. As far as we know this strategy has not been applied to the context of parallel machines. In this paper we investigate the computational impact of using the SCF constraints rather than the MTZ constraints in the CLSD and CLSD ${ }^{w}$ models for the LSP-PLSR.

To define the CLSD $\mathrm{SCF}_{\text {and }}$ CLSD $\mathrm{SCF}^{w}$ models, for each production line and period, we identify the products as nodes in a complete graph. In this graph, only the nodes associated with the products produced in the respective line and period are visited. Therefore, a production sequence consists of a simple path that contains only the nodes that are associated with the produced items. Suppose that at the beginning of each period there is an amount of a general commodity available (this quantity needs to be equal to the number of different items that are produced in the current period and line) and, for each visited node, exactly one unit of the commodity needs to be left at the node. At the end of the period, the final balance of the commodity needs to be equal to zero. An artificial node indexed by 0 is introduced acting as the source of the flow, i.e., the initial amount of the commodity comes from the artificial node. In this model, the auxiliary variables $V_{l j t}$ become $V_{l i j t}$ to indicate the amount of the commodity that is transferred on line $l$ from node $i$ to $j$ in period $t$.

The CLSD $\mathrm{SCF}_{\text {and }}$ CLSD $\mathrm{SCF}^{w}$ models can be obtained from the CLSD and CLSD ${ }^{w}$ models, respectively, using constraints (3.47), (3.48), (3.49), and (3.50) rather than the MTZ constraints (3.14).

$$
\begin{array}{ll}
\sum_{j \in P_{l}} V_{l 0 j t}=\sum_{j \in P_{l}} w_{l j t}, & \forall l, t \\
V_{l 0 j t} \leq \gamma_{l t} y_{l j t}, & \forall l, j, t \\
\sum_{i \in P_{l} \cup\{0\}} V_{l i j t}-\sum_{i \in P_{l}} V_{l j i t}=y_{l j t}+\sum_{i \in P_{l}} z_{l j i t}, & \forall l, j \in P_{l}, t \\
V_{l i j t} \leq\left(\gamma_{l t}-1\right) z_{l i j t}, & \forall l, i, j \in P_{l}, t \\
V_{l i j t} \geq 0 . & \forall l, i, j, t
\end{array}
$$

For each line, constraints (3.47) ensure that the amount of commodity available at the beginning of each period is exactly the number of items to be produced. Given the minimum lot size imposed by constraints (3.45) this information is captured through the variables $w_{l j t}$ in the RHS of (3.47). Constraints (3.48) ensure that the commodity from the artificial node can only be transferred to the node associated to the first item produced in line $l$. Constraints (3.49) controls the balance of the commodity at the nodes, i.e., the quantity of commodity that leaves each visited node is equal to the quantity that enters node minus one. Constraints (3.50) ensure that commodity flow only occurs between nodes visited consecutively. Finally, constraints (3.51) define the domain of the variables $V_{l i j t}$. 
Summary of the models $C L S D_{\mathrm{SCF}}$ and $C L S D_{\mathrm{SCF}}^{w}$ for the LSP-PLSR

$$
\begin{aligned}
\text { CLSD }_{\mathrm{SCF}}: & \text { Min (3.2) } & \text { s.t.: } & (3.4)-(3.13),(3.16)-(3.23),(3.47)- \\
& & & (3.50) \text { and }(3.5)-(3.25) \\
\text { CLSD }_{\mathrm{SCF}}^{w}: & \operatorname{Min}(3.2) & \text { s.t.: } & (3.4),(3.7),(3.45),(3.12),(3.13),(3.46), \\
& & & (3.21)-(3.51) \text { and }(3.5)-(3.25)
\end{aligned}
$$

\subsection{2. $F L-C L S D^{w}$ and $F L-C L S D_{\mathrm{SCF}}^{w}$ models}

In Section 3.4.1 we presented the facility location reformulation for the GLSPCC model. In this section, we describe the facility location reformulation for the CLSD ${ }^{w}$ and CLSD $_{\mathrm{SCF}}^{w}$ models (denominated FL-CLSD ${ }^{w}$ model and FL-CLSD ${ }_{\mathrm{SCF}}^{w}$ model, respectively).

Let $x_{l j t t^{\prime}}$ be the amount of item $j$ produced on line $l$ in period $t$ in order to meet the demands due to period $t^{\prime}$. The objective function (3.2) and constraints (3.4), (3.7) and (3.45) need to be reformulated as follows.

- The objective function (3.2) is adapted according to the redefinition of the production variables, i.e., we use the objective function (3.52) instead (3.2).

$$
\operatorname{Min} \sum_{l, j, t, t^{\prime}} h c_{l j t t^{\prime}} x_{l j t t^{\prime}}+\sum_{l, i, j, t} s c_{l i j} z_{l i j t}+\sum_{l, t} a c_{l} \delta_{l t} .
$$

- The inventory balance constraints (3.4) are replaced by constraints (3.53).

$$
\sum_{l} \sum_{t^{\prime}} x_{l j t^{\prime} t}=d_{j t} . \quad \forall j, t
$$

- The capacity constraints (3.7) are slightly modified to consider the redefinition of the production variables as described in (3.54).

$$
\sum_{j \in P_{l}} \sum_{t^{\prime}} a_{l j} x_{l j t t^{\prime}}+\sum_{i, j \in P_{l}} s t_{l i j} z_{l i j t} \leq C_{l t} . \quad \forall l, t
$$

- The setup state and minimum lot sizes constraints (3.45) also need to be updated by (3.55).

$$
m_{j} w_{l j t} \leq \sum_{t^{\prime}} x_{l j t t^{\prime}} \leq \frac{C_{l t}}{a_{j}} w_{l j t} . \quad \forall l, j, t
$$

- Constraints (3.56) define the domain of the new variables.

$$
x_{l j t t^{\prime}} \geq 0 . \quad \forall l, j, t, t^{\prime}
$$

3.7.3. Summary of the models $F L-C L S D^{w}$ and $F L-C L S D_{\mathrm{SCF}}^{w}$ for the $L S P-P L S R$

$$
\begin{array}{rlrl}
\text { FL-CLSD }^{w}: & \text { Min (3.52) } & \text { s.t.: } & (3.53),(3.54),(3.55),(3.12),(3.13), \\
& & (3.14),(3.46),(3.56),(3.22)-(3.44), \\
& (3.5),(3.17), \text { and }(3.25) \\
& & (3.53),(3.54),(3.55),(3.12),(3.13), \\
\text { FL-CLSD }_{\text {SCF }}^{w}: \quad \text { Min (3.52) } & \text { s.t.: } 3.46),(3.56),(3.22)-(3.51), \\
& & (3.5),(3.17), \text { and }(3.25)
\end{array}
$$

\subsection{The main characteristics of the proposed models}

In Sections 3.1, 3.3, and 3.6 we have proposed nine MIP models to represent the LSP-PLSR. In general, the proposed models consist of extensions of models presented in the literature for the traditional LSP to consider the decisions regarding organizing production lines taking into account the availability of scarce resources. In this section, we summarize and analyze the main characteristics of the proposed models. 
TABLE 3. Summarizing the proposed models. Best values are marked in bold.

\begin{tabular}{|c|c|c|c|c|c|}
\hline Model & $\mathrm{CV}$ & BV & $\mathrm{SD}$ & $\mathrm{SU}$ & $\mathrm{RB}$ \\
\hline GLSP & $\mathrm{LJ} \gamma \mathrm{T}+\mathrm{LJ}^{2} \gamma \mathrm{T}+2 \mathrm{JT}$ & $\mathbf{L} \mathbf{J} \gamma \mathbf{T}$ & MP & $\mathrm{E}$ & {$[9,21]$} \\
\hline GLSP $^{\mathrm{CC}}$ & $\mathrm{LJ} \gamma \mathrm{T}+2 \mathrm{JT}$ & $\mathrm{LJ}^{2} \gamma \mathrm{T}+\mathrm{LT}$ & MP & I & {$[5,21]$} \\
\hline FL-GLSP ${ }^{C C}$ & $\mathrm{LJ}_{\gamma} \mathrm{T}^{2}$ & $\mathrm{LJ}^{2} \gamma \mathrm{T}+\mathrm{LT}$ & MP & I & {$[5,8,21]$} \\
\hline CLSD & $2 \mathrm{LJT}+2 \mathrm{JT}$ & $\mathrm{LJT}+\mathrm{LJ}^{2} \mathrm{~T}+\mathrm{LT}$ & MTZ & I & [15] \\
\hline CLSD $^{w}$ & $2 \mathrm{LJT}+2 \mathrm{JT}$ & $2 \mathrm{LJT}+\mathrm{LJ}^{2} \mathrm{~T}+\mathrm{LT}$ & MTZ & $\mathrm{E}$ & {$[15,23]$} \\
\hline FL-CLSD $^{w}$ & $\mathrm{LJT}^{2}+\mathrm{LJT}$ & $2 \mathrm{LJT}+\mathrm{LJ}^{2} \mathrm{~T}+\mathrm{LT}$ & MTZ & $\mathrm{E}$ & {$[8,15,23]$} \\
\hline CLSD $_{\mathrm{SCF}}$ & $\mathrm{LJ}^{2} \mathrm{~T}+\mathrm{LJT}+2 \mathrm{JT}$ & $\mathrm{LJT}+\mathrm{LJ}^{2} \mathrm{~T}+\mathrm{LT}$ & $\mathrm{SCF}$ & I & {$[11,13,15]$} \\
\hline $\mathrm{CLSD}_{\mathrm{SCF}}^{w}$ & $\mathrm{LJT}+2 \mathrm{JT}+\mathrm{LJ}^{2} \mathrm{~T}$ & $2 \mathrm{LJT}+\mathrm{LJ}^{2} \mathrm{~T}+\mathrm{LT}$ & $\mathrm{SCF}$ & $\mathrm{E}$ & $\begin{array}{l}{[15,23]} \\
{[11,13]}\end{array}$ \\
\hline FL-CLSD ${ }_{\mathrm{SCF}}^{w}$ & $\mathrm{LJT}^{2}+\mathrm{LJ}^{2} \mathrm{~T}$ & $2 \mathrm{LJT}+\mathrm{LJ}^{2} \mathrm{~T}+\mathrm{LT}$ & $\mathrm{SCF}$ & $\mathrm{E}$ & $\begin{array}{l}{[11,15,23],} \\
{[8,13]}\end{array}$ \\
\hline
\end{tabular}

Table 3 presents, for each model, the total number of continuous (CV) and binary (BV) variables as functions of the parameters described in Table 1. It also shows, for each proposed model, the strategy used to model the sequencing decisions (column SD), the explicit use (E) or not (I) of binary variables to model the setup states of the lines (column SU), and the references that served as the basis for each proposed model (column RB). In the SD column, MP indicates the utilization of micro periods as the sequencing strategy, while MTZ (or SCF) indicates that the structure of the travelling salesman problem was used to model the sequencing decisions and the type of constraints that are used to eliminate subsequences. For simplicity, we have considered that each product can be produced on every production line and that each production period is composed of $\gamma$ micro periods for all production lines.

Since $2 \leq \gamma \leq J$, we can see that the GLSP model uses the smallest number of binary variables, while the CLSD and CLSD ${ }^{w}$ models use the smallest numbers of continuous variables (marked in bold in Table 3). On the other hand, the GLSP ${ }^{C C}$ and FL-GLSPCC models use the largest number of binary variables (considering the values that will be presented in Sect. 4.1) and the GLSP model uses the largest number of continuous variables.

The computational results presented in Section 4 shows that the number of binary and continuous variables present in a model is not the only factor that explains its computational performance. Models with the smallest number of variables (binary and/or continuous) do not necessarily present the best results. Other elements of the modeling approach have a greater influence on the computational behaviour of the models.

Finally, we note that the nine models listed in Table 3 do not cover all possible combinations among the modeling strategies analysed in this work. More specifically, we have excluded three models: FL-GLSP, FL-CLSD and FL-CLSD ${ }_{\mathrm{SCF}}$. In a preliminary computational experiment we observed that the GLSPCC ${ }^{C}$, the CLSD $^{w}$ and

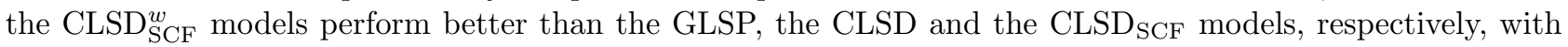
respect to the ability to find feasible solutions and/or to the quality of the solutions found. Hence, we have only considered the facility location reformulation for the more promising models with respect to the first criterion analysed (explicit use of binary variables to model the setup states of the lines).

\section{Computational Results}

The nine mathematical optimization models summarized in Table 3 were implemented in the $\mathrm{C}++$ language using the Concert Technology library of the Cplex 12.6 solver. The instances described in Section 4.1 were solved by the branch-and-cut algorithm of the Cplex solver using default settings and with the maximum running time fixed at 3600 seconds (i.e., an hour). They were executed on a computer with two Intel Xeon $2.8 \mathrm{GHz}$ processors and 128 GB DDR3 RAM memory. 
TABLE 4. Characteristics of the five classes of instances.

\begin{tabular}{lllllllllll}
\hline \hline Class & $|T|$ & $|L|$ & $|J|$ & $|K|$ & $\varphi^{d}$ & $\varphi_{k}^{r}$ & $\varphi_{0}^{r}$ & $\phi^{b}$ & $\phi^{e}$ & $\gamma$ \\
\hline 1 & 10 & 7 & 45 & 5 & 100 & 0.8 & 0.6 & 0 & 0 & 6 \\
2 & 10 & 10 & 80 & 6 & 100 & 0.8 & 0.6 & 0 & 0 & 8 \\
3 & 14 & 10 & 90 & 6 & 90 & 0.6 & 0.5 & 0 & 0 & 8 \\
4 & 12 & 10 & 110 & 7 & 90 & 0.6 & 0.55 & 100 & 150 & 8 \\
5 & 14 & 10 & 110 & 7 & 90 & 0.6 & 0.55 & 50 & 150 & 8 \\
\hline
\end{tabular}

\subsection{Main features of the instances}

Two sets of instances were used to evaluate the computational performance of the proposed models. One set is composed of 100 random generated instances divided into five classes as proposed in [24]. The data used to generate these instances are based on the parameter settings observed in some food industries. In this paper, we denote these 100 instances as the default data set and use them to study the main properties of the nine models proposed in Section 3.

For this set of instances, the number of periods $|T|$ belongs to the set $\{10,12,14\}$, the number of production lines $|L|$ to the set $\{7,10\}$, and the product catalogue are composed by $|J| \in\{45,80,90,110\}$ products. The number of considered resources $|K|$ ranges from 5 to 7 . The other parameters were generated using uniform distributions (indicated as $p \in U[a, b]$ ) as described next. Table 4 shows the specific parameter values adopted for each class of instances. A detailed description about the parameter values adopted in the default data set can be found in [24].

- Demands: $d_{j t} \in U\left[0, \frac{{ }_{{ }_{l t}-} \min _{i, j}\left\{s t_{l i j}\right\} \gamma-\varphi^{d}}{\left|P_{l}\right|}\right]$, with $\varphi^{d}$ as specified in Table 4 .

- Production capacity: $C_{l t}=480$ (minutes in a production day of eight hours);

- Unit processing times: $a_{l j}=1$;

- Minimum lot sizes: $m_{l j}=2$;

- Inventory holding and backlogging costs: $h_{j} \in U[1,10]$ and $b_{j}=10 h_{j}$;

- Setup times and costs: $s t_{l i j} \in U[15,45]$ and $s c_{l i j}=2 s t_{l i j}$;

- Amount of each resource necessary to organize the lines: $r_{k l} \in U[0,2]$ when $k>0$ and $r_{0 l} \in U[5,10]$. In [24], the authors have distinguished the resource $k=0$ from the others $(k>0)$. More specifically, they considered that $k=0$ represents the workers, while $k=1, \cdots,|K|-1$ represent various types of machines as illustrated in Figure 1;

- Capacity of the production resources: $R_{k t}=\max \left\{\max _{l=1, \ldots, L}\left\{r_{k l}\right\}, \varphi_{k}^{r} \sum_{l \in[L]} r_{k l}\right\}$, with $\varphi_{k}^{r}$ as specified in Table 4 . We note that the parameters $\varphi_{k}^{r}$ define the maximum percentage of production lines that can operate simultaneously;

- Costs to organize the lines: $a c_{l}=\sum_{k} r c_{k} r_{k l}$, where $r c_{k} \in U\left[\phi^{b}, \phi^{e}\right]$ with $\phi^{b}$ and $\phi^{e}$ as specified in Table 4 . The parameter $r c_{k}$ represents the unit price of resource $k$;

- Maximum number of different products that can be produced on each line and period: $\gamma_{l t}=\gamma$, with $\gamma$ as specified in Table 4;

- Assignment of products to the lines: For each product, there is only one production line able to produce it. This line was randomly selected.

The second set of instances is used to perform a sensitivity analysis with respect to the parameter $\varphi_{k}^{r}$ that differentiates the LSP-PLSR from the traditional LSP. If $\varphi_{k}^{r}=1, \forall k$, we obtain the traditional LSP on 
parallel machines in which all production lines operate during all periods. Moreover, the lower the values of the parameters $\varphi_{k}^{r}$, the fewer the number of lines that can operate simultaneously.

The instances in the default data set were generated using values for the parameter $\varphi_{k}^{r}$ in line with the specific application addressed in [24]. In order to fully evaluate the impact of this parameter, we perform a sensitivity analysis considering five different values. By setting $\varphi_{k}^{r}=\varphi, \forall k$, we have successively fixed the value of the parameter $\varphi$ to $0.6 ; 0.7 ; 0.8 ; 0.9$; and 1 ; to obtain, for each value, 100 instances. The other parameters used are the ones shown in Table 4. So, a total of 500 instances make up the second set of instances that is used in the sensitivity analysis described in Section 4.4.

\subsection{Analysis of the modeling strategies}

In this section, we present the results of a computational experiment designed to study the different modeling strategies proposed in Section 3 for the LSP-PLSR. More specifically, in this section, we analyze the computational impact of the following aspects:

- Explicit binary production variables (Sect. 4.2.1). The models that explicit the zero-or-one variables to indicate if the products are or are not produced on each line in each period (variables $w_{l j s}$ in the GLSP and $w_{l j t}$ in the CLSD based models) have a greater number of binary variables. However, sometimes, this strategy can improve the computational performance of the model as discussed in [23];

- Sequencing strategy (Sect. 4.2.2). We compare the performance of the MP, MTZ, and SCF strategies used to model the sequencing decisions;

- Facility location reformulation (Sect. 4.2.3). We study the efficiency of the facility location reformulation combined with other modeling strategies.

Our analysis is based on Table 5 which shows the number of instances in which at least one feasible solution was found (FS), the number of instances in which the optimal solution (OS) was obtained, the average objective function value $(\mathrm{OF})$, the average dual bound $(\mathrm{DB})$, the average GAP (AG), the best GAP (BG), the worst GAP (WG), and the average running time (RT). The best values obtained are highlighted in bold. In this Table, the GAPs (percentage deviation of OF from DB) were computed as GAP $=100 * \frac{\mathrm{FO}-\mathrm{DB}}{\mathrm{FO}}$.

\subsubsection{Explicit production binary variables}

Note that, in the GLSP model, the binary production variables $\left(w_{l j s}\right)$ are explicit while, in the GLSPCC model, these variables are implicit. Table 5 shows that, for classes 1 and 2, the GLSP provided better feasible solutions than the GLSP ${ }^{C C}$ while the GLSP ${ }^{C C}$ provided better dual bounds than the GLSP. On average, the GAPs obtained by the GLSPCC were smaller than those obtained by the GLSP for these classes. However, we also note that, the GLSP model was not able to find feasible solutions for 34 instances (11 being from class 3, 11 from class 4 and 12 from class 5), while GLSP ${ }^{C C}$ found feasible solutions for all instances. Therefore, we conclude that the model in which the production binary variables $\left(w_{l j s}\right)$ are considered implicitly performs better than the model that gives them explicit, i.e., defining the production binary variables explicitly is not a good approach when the micro period (MP) strategy is used to model the sequencing decisions.

On the other hand, analyzing the performance of the CLSD and CLSD ${ }^{w}$ models, we can see that, the CLSD ${ }^{w}$ model produced better results for instances from classes 1, 2, 3 and 4, providing better feasible solutions, dual bounds and GAPs. Moreover, for classes 1 and 2, the CLSD ${ }^{w}$ model found optimal solutions for more instances and had significantly shorter running times. Although the CLSD ${ }^{w}$ model provided better dual bounds for instances from class 5, the CLSD model provided significantly better feasible solutions, resulting in smaller GAPs for this class and significantly impacting the average performance. In general, we can conclude that expliciting the binary production variables was a good strategy for the CLSD based models using the MTZ sequence strategy as is highlighted in the performance profile analysis discussed in Section 4.3.

Finally, considering the CLSD SCF $_{\text {and }}$ CLSD $_{\mathrm{SCF}}^{w}$ models, we can see that the CLSD $\mathrm{SCF}^{w}$ model outperforms the CLSD $_{\mathrm{SCF}}$ model with respect to the quality of the feasible solutions and dual bounds obtained (and consequently with respect to the GAPs) for all classes. Therefore, defining the binary production variables was a good strategy for the models using the SCF sequence strategy. 
TABLE 5. Results obtained by the proposed models. Best values are marked in bold.

\begin{tabular}{|c|c|c|c|c|c|c|c|c|c|}
\hline Class & Model & FS & OS & $\mathrm{OF}$ & DB & $\mathrm{AG}$ & $\mathrm{BG}$ & WG & $\mathrm{RT}$ \\
\hline \multirow[t]{9}{*}{1} & GLSP & 20 & 0 & 51869 & 41658 & 19.90 & 14.80 & 26.57 & 3600 \\
\hline & $\mathrm{GLSP}^{\mathrm{CC}}$ & 20 & 0 & 52086 & 46068 & 11.22 & 4.70 & 19.36 & 3600 \\
\hline & FL-GLSP CC & 20 & 0 & 51559 & 46415 & 9.95 & 1.17 & 19.42 & 3600 \\
\hline & CLSD & 20 & 5 & 50923 & 50824 & 0.18 & 0.00 & 0.72 & 2972 \\
\hline & $\mathrm{CLSD}^{w}$ & 20 & 18 & 50921 & 50914 & 0.01 & 0.00 & 0.05 & 1011 \\
\hline & FL-CLSD $^{w}$ & 20 & 14 & 50921 & 50911 & 0.02 & 0.00 & 0.06 & 1212 \\
\hline & CLSD $_{\mathrm{SCF}}$ & 20 & 16 & 50921 & 50878 & 0.07 & 0.00 & 0.44 & 1448 \\
\hline & $\mathrm{CLSD}_{\mathrm{SCF}}^{w}$ & 20 & 20 & 50921 & 50921 & 0.00 & 0.00 & 0.00 & 136 \\
\hline & FL-CLSD ${ }_{\mathrm{SCF}}^{w}$ & 20 & 20 & 50921 & 50921 & 0.00 & 0.00 & 0.00 & 77 \\
\hline \multirow[t]{9}{*}{2} & GLSP & 20 & 0 & 84131 & 41525 & 50.19 & 40.26 & 60.26 & 3600 \\
\hline & GLSP $^{\mathrm{CC}}$ & 20 & 0 & 93359 & 44574 & 49.10 & 35.29 & 74.97 & 3600 \\
\hline & FL-GLSP ${ }^{C C}$ & 20 & 0 & 75171 & 48503 & 35.08 & 21.26 & 51.19 & 3600 \\
\hline & CLSD & 20 & 0 & 63652 & 62647 & 1.58 & 0.18 & 3.71 & 3600 \\
\hline & $\mathrm{CLSD}^{w}$ & 20 & 2 & 63595 & 63554 & 0.07 & 0.00 & 0.12 & 3402 \\
\hline & FL-CLSD $^{w}$ & 20 & 2 & 63595 & 63548 & 0.08 & 0.00 & 0.22 & 3361 \\
\hline & $\mathrm{CLSD}_{\mathrm{SCF}}$ & 20 & 3 & 63607 & 63466 & 0.22 & 0.00 & 0.66 & 3322 \\
\hline & $\mathrm{CLSD}_{\mathrm{SCF}}^{w}$ & 20 & 17 & 63596 & 63503 & 0.15 & 0.00 & 1.09 & 1439 \\
\hline & FL-CLSD ${ }_{\mathrm{SCF}}^{w}$ & 20 & 20 & 63595 & 63589 & 0.00 & 0.00 & 0.00 & 427 \\
\hline \multirow[t]{9}{*}{3} & GLSP & 9 & 0 & 666242 & 58613 & 89.34 & 84.21 & 95.72 & 3600 \\
\hline & GLSP $^{C C}$ & 20 & 0 & 1194620 & 74258 & 93.08 & 84.21 & 95.90 & 3600 \\
\hline & FL-GLSP ${ }^{C C}$ & 2 & 0 & 697921 & 95261 & 83.34 & 76.66 & 90.02 & 3600 \\
\hline & CLSD & 20 & 0 & 181857 & 113026 & 33.26 & 12.49 & 65.12 & 3600 \\
\hline & $\mathrm{CLSD}^{w}$ & 20 & 0 & 160403 & 115590 & 24.85 & 7.23 & 54.65 & 3600 \\
\hline & FL-CLSD $^{w}$ & 20 & 0 & 307369 & 115553 & 50.14 & 13.65 & 83.81 & 3600 \\
\hline & $\mathrm{CLSD}_{\mathrm{SCF}}$ & 20 & 0 & 147747 & 113392 & 21.59 & 9.05 & 42.77 & 3600 \\
\hline & CLSD $_{\mathrm{SCF}}^{w}$ & 20 & 0 & 140899 & 113784 & 17.88 & 3.41 & 39.23 & 3600 \\
\hline & FL-CLSD ${ }_{\mathrm{SCF}}^{w}$ & 20 & 0 & 136481 & 116422 & 13.50 & 0.03 & 37.97 & 3600 \\
\hline \multirow[t]{9}{*}{4} & GLSP & 9 & 0 & 746464 & 134924 & 76.52 & 50.53 & 90.10 & 3600 \\
\hline & $\mathrm{GLSP}^{\mathrm{CC}}$ & 20 & 0 & 1000956 & 137739 & 85.79 & 82.17 & 89.70 & 3600 \\
\hline & FL-GLSP ${ }^{C C}$ & 0 & 0 & - & - & - & - & - & - \\
\hline & CLSD & 20 & 0 & 366638 & 172700 & 33.26 & 13.44 & 72.00 & 3600 \\
\hline & $\mathrm{CLSD}^{w}$ & 20 & 0 & 289695 & 175532 & 28.88 & 9.72 & 76.89 & 3600 \\
\hline & FL-CLSD $^{w}$ & 18 & 0 & 408052 & 176497 & 47.76 & 29.74 & 71.25 & 3600 \\
\hline & CLSD $_{\mathrm{SCF}}$ & 20 & 0 & 214687 & 173688 & 18.71 & 9.37 & 35.29 & 3600 \\
\hline & $\mathrm{CLSD}_{\mathrm{SCF}}^{w}$ & 20 & 0 & 203052 & 174327 & 14.02 & 6.29 & 22.04 & 3600 \\
\hline & FL-CLSD ${ }_{\mathrm{SCF}}^{w}$ & 20 & 0 & 196133 & 177612 & 9.36 & 1.96 & 17.20 & 3600 \\
\hline \multirow[t]{9}{*}{5} & GLSP & 8 & 0 & 1161164 & 159855 & 84.41 & 73.41 & 91.29 & 3600 \\
\hline & GLSP $^{\mathrm{CC}}$ & 20 & 0 & 1148319 & 163438 & 85.61 & 80.87 & 89.07 & 3600 \\
\hline & FL-GLSPCC & 0 & 0 & - & - & - & - & - & - \\
\hline & CLSD & 20 & 0 & 474437 & 209923 & 54.67 & 41.21 & 66.70 & 3600 \\
\hline & $\mathrm{CLSD}^{w}$ & 20 & 0 & 628712 & 213629 & 61.18 & 31.31 & 81.84 & 3600 \\
\hline & FL-CLSD $^{w}$ & 18 & 0 & 542487 & 215164 & 55.18 & 25.80 & 78.66 & 3600 \\
\hline & CLSD $_{\mathrm{SCF}}$ & 20 & 0 & 299022 & 210017 & 20.38 & 9.70 & 82.19 & 3600 \\
\hline & $\mathrm{CLSD}_{\mathrm{SCF}}^{w}$ & 20 & 0 & 250429 & 211416 & 15.30 & 8.79 & 21.43 & 3600 \\
\hline & FL-CLSD ${ }_{\mathrm{SCF}}^{w}$ & 20 & 0 & 242944 & 215031 & 11.07 & 4.78 & 23.43 & 3600 \\
\hline \multirow[t]{9}{*}{ Mean } & GLSP & 13.2 & 0 & 541974 & 87315 & 64.07 & 52.64 & 72.79 & 3600 \\
\hline & $\mathrm{GLSP}^{\mathrm{CC}}$ & 20 & 0 & 697868 & 93215 & 64.96 & 57.45 & 73.80 & 3600 \\
\hline & FL-GLSPCC & 8.4 & 0 & - & - & - & - & - & - \\
\hline & CLSD & 20 & 1 & 227501 & 121824 & 24.59 & 13.46 & 41.65 & 3474 \\
\hline & $\mathrm{CLSD}^{w}$ & 20 & 4 & 238665 & 123844 & 23.00 & 9.65 & 42.71 & 3043 \\
\hline & FL-CLSD $^{w}$ & 19.2 & 3.2 & 274485 & 124335 & 30.63 & 13.84 & 46.80 & 3075 \\
\hline & $\mathrm{CLSD}_{\mathrm{SCF}}$ & 20 & 3.8 & 155197 & 122288 & 12.19 & 5.62 & 32.27 & 3114 \\
\hline & $\mathrm{CLSD}_{\mathrm{SCF}}^{w}$ & 20 & 7.4 & 141779 & 122789 & 9.47 & 3.70 & 16.76 & 2475 \\
\hline & FL-CLSD ${ }_{\mathrm{SCF}}^{w}$ & 20 & 8 & 138015 & 124714 & 6.79 & 1.35 & 15.72 & 2261 \\
\hline
\end{tabular}




\subsubsection{Sequencing strategy analysis}

Through the results presented on Table 5, we can easily see that the models using the MP strategy (GLSP, GLSP $^{C C}$, and FL-GLSPCC ) can-not overcome the performance of the other models (CLSD, CLSD ${ }^{w}$, FL-CLSD $^{w}$, $\mathrm{CLSD}_{\mathrm{SCF}} \mathrm{CLSD}_{\mathrm{SCF}}^{w}$, and FL-CLSD ${ }_{\mathrm{SCF}}$ ) for all classes of instances with respect to all the benchmarks being considered (feasible solutions, dual bounds, GAPs and running times). Moreover, the models using the SCF strategy provided in general better results than the models using the MTZ strategy. More specifically, the SCF models were able to deliver better feasible solutions, GAPs and running times. The SCF models were also able to find optimal solutions for a larger number of instances. With respect to the dual bounds, we observed that the impact of the adopted sequence strategy depends on other modeling aspects. For example, the MTZ models that use explicit the binary production variables $\left(\mathrm{CLSD}^{w}\right.$ and FL-CLSD ${ }^{w}$ ) provided better dual bounds than the $\mathrm{CLSD}_{\mathrm{SCF}}$ and CLSD $\mathrm{SCF}^{w}$ models. However, they were not able to provide better dual bounds than the FL-CLSD ${ }_{\mathrm{SCF}}^{w}$ model. In general, considering the GAPs and running times obtained, we can conclude that SCF was the best strategy to model the sequencing decisions for the LSP-PLSR.

\subsubsection{Facility location reformulation analysis}

Analyzing the performance of the GLSPCC and FL-GLSP ${ }^{C C}$ models, Table 5 shows that the FL-GLSPCC model presented better results than the GLSPCC model only for classes 1 and 2. For classes 3,4 and 5 , the FL-GLSP ${ }^{C C}$ model was not able to provide feasible solutions for most instances, while GLSP ${ }^{C C}$ provided feasible solutions for all instances.

Among the models using the MTZ strategy, the impact of the FL reformulation can be evaluated comparing the performance of the CLSD ${ }^{w}$ and FL-CLSD ${ }^{w}$ models. These models presented very similar feasible solutions for classes 1 and 2, but the CLSD ${ }^{w}$ model presented significantly better feasible solutions for classes 3 and 4 . The OF value obtained by the FL-CLSD ${ }^{w}$ model for class 5 was smaller than the value obtained by the CLSD ${ }^{w}$ model. However, this fact occurred because FL-CLSD ${ }^{w}$ was not able to find feasible solutions for two difficult instances from class 5 . On the other hand, FL-CLSD ${ }^{w}$ was able to provide slightly better dual bounds for all classes. In general, we can conclude that CLSD $^{w}$ performed better than the FL-CLSD ${ }^{w}$ model.

Finally, in order to evaluate the FL reformulation of the models using the SCF strategy, we have compared the performance of the $\mathrm{CLSD}_{\mathrm{SCF}}^{w}$ and FL-CLSD $\mathrm{SCF}^{w}$ models. We can easily see that FL-CLSD $\mathrm{SCF}_{\text {provided }}^{w}$ better results than CLSD $_{\mathrm{SCF}}^{w}$ for all classes (although these models provided the same values for OF, DB, and GAPs for the instances in class 1, FL-CLSD $\mathrm{SCF}^{w}$ presented a smaller RT for this class). Therefore, the FL reformulation was able to significantly improve the computational performance of the models using the SCF sequencing strategy, while for the models using the MP and MTZ strategies, the FL reformulation was not a good strategy.

\subsection{Performance analysis of the models}

Comparing the proposed models with respect to their ability to find high quality feasible solutions, Figure 2 shows the performance profile as proposed in [7]. For each instance $\alpha$ and model $\beta$, let $z_{\alpha}^{\beta}$ be the OF value obtained by model $\beta$ for instance $\alpha$, and let $\tau_{\alpha}=\min _{\beta}\left\{z_{\alpha}^{\beta}\right\}$ be the minimum OF value obtained by instance $\alpha$. We denote the quotient between $z_{\alpha}^{\beta}$ and $\tau_{\alpha}$ by $\Gamma_{\alpha}^{\beta}$, i.e., $\Gamma_{\alpha}^{\beta}$ is the relative deviation of the OF value obtained by model $\beta$ from the best OF value obtained for instance $\alpha$. In Figure 2, the $\mathrm{x}$-axis presents the relative deviations $(\theta)$ and the $\mathrm{y}$-axis presents the percentage of instances for which $\Gamma_{\alpha}^{\beta} \leq \theta$. For example, when $\theta=1$ on the $\mathrm{x}$-axis, the $\mathrm{y}$-axis presents the percentage of instances for which each model provided the best OF value.

According to the curves presented on Figure 2, we can see that the FL-CLSD SCF $^{w}$ model obtained the smallest OF value for 88 instances and obtained the smallest maximum deviation (about 1.13) from the best OF value. The CLSD ${ }_{\mathrm{SCF}}^{w}$ model provided the smallest OF value for 45 instances and CLSD ${ }_{\mathrm{SCF}}$ for 41 instances. The maximum deviation from the best solution for these models was 1.26 and 4.49, respectively. Therefore, the FL-CLSD ${ }_{\mathrm{SCF}}^{w}$ model performs significantly better than the others. 


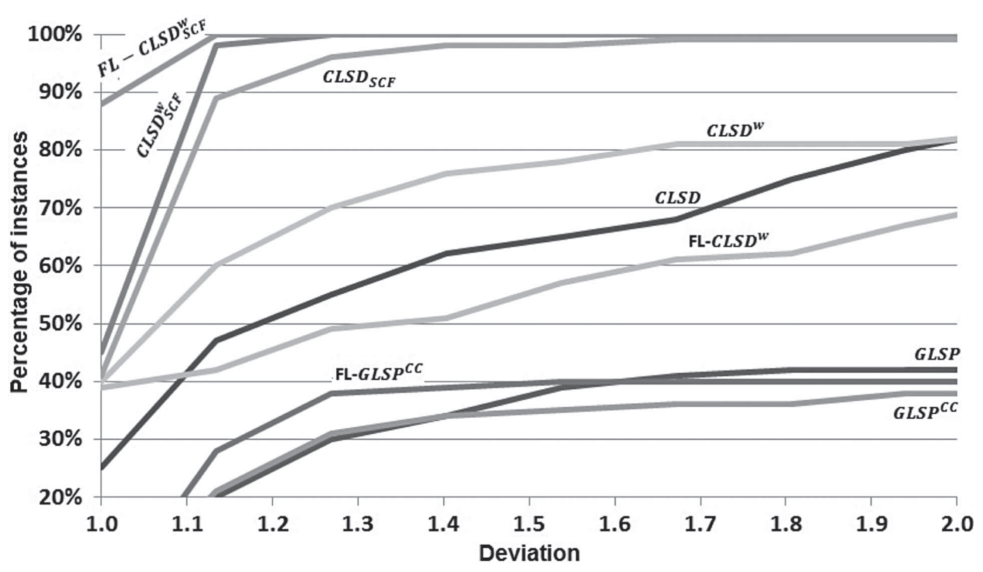

FiguRE 2. Performance of the proposed models.

We can also see that the CLSD $^{w}$ model provided smallest OF values for 40 instances, FL-CLSD ${ }^{w}$ for 39 instances and CLSD for 25 instances. Moreover, we observe that the FL-CLSD ${ }^{w}$ model was not able to find good feasible solutions for instances from classes 3, 4, and 5, presenting (in general) higher maximum deviations than the CLSD and CLSD ${ }^{w}$ models. Therefore, the facility location reformulation was not able to improve the performance of the MTZ based models for large sized instances (classes 3, 4, and 5).

The models using the MP sequence strategy were not able to find the best OF value for any instance. Moreover, these models presented very similar computational performances. Among these models, GLSPCC stands out due to its ability to find feasible solutions for all instances.

\subsection{Sensitivity analysis}

In this section, we study the impact of varying the values of the parameters that define the proportion of production lines that can simultaneously operate in each period. Two aspects are considered: (i) the computational difficulty to solve the problem, and (ii) the structure of the solutions obtained. Since the FL-CLSD ${ }_{\mathrm{SCF}}^{w}$ model was identified as the most promising formulation for the LSP-PLSR (Sects. 4.2 and 4.3), only this model is considered in this sensitivity analysis. This analysis was carried out using the 500 instances from the second data set proposed in Section 4.1 obtained by setting $\varphi_{k}^{r}=\varphi, \forall k$, with $\varphi$ equals to $1 ; 0.9 ; 0.8$; 0.7 ; and 0.6 . The results are shown in Table 6 where the first 6 columns show the results for the instances in Classes 1, 3 and 5 and the last 6 show the results for the ones in Classes 2 and 4 and the average values (Mean).

From the results presented in Table 6 we can easily see that, for all classes, the traditional $\operatorname{LSP}(\varphi=1)$ is much easier to solve than the LSP-PLSR $(\varphi<1)$. Moreover, the running times and/or GAPs obtained significantly increased when the value of the parameter $\varphi$ decreased, indicating that the problem becomes more challenging from the computational perspective as the number of production lines that can simultaneously operate in each period reduces. We also observed that the OF values significantly increased when $\varphi$ decreased. In order to identify the changes in the structure of the solutions, in Figures 3 and 4 we detailed the OF values presenting: the inventory holding (IC), the backlogging (BC), the setup (SC) costs, and the cost of organizing the production lines $(\mathrm{OC})$.

For classes 1, 2 and 3 (Fig. 3), we can see that when the value of the parameter $\varphi$ increased, the inventory and backlogging costs significantly decreased, while the setup costs increased. For classes 4 and 5 (Fig. 4), the same fact was observed, however, in these cases the costs to organize the lines also significantly increased. Note that as the value of the parameter $\varphi$ increases, more production lines can operate, and so, more setups occur for each item during the planning horizon, backlogging is avoided and the size of the production lots is reduced. As a result, the setup and/or organizing costs increase and the inventory and backlogging costs decrease. 
TABLE 6. Sensitivity analysis with respect to the parameter $\varphi$.

\begin{tabular}{l|l|llll|l|llll}
\hline \hline Class & $\varphi$ & OF & DB & AG & RT & Class & OF & DB & AG & RT \\
\hline \multirow{2}{*}{1} & 1 & 16244 & 16244 & 0.00 & 7 & 2 & 25129 & 25128 & 0.01 & 26 \\
& 0.9 & 30219 & 30219 & 0.00 & 21 & & 40730 & 40727 & 0.01 & 94 \\
& 0.8 & 39510 & 39510 & 0.00 & 44 & & 51980 & 51946 & 0.06 & 408 \\
& 0.7 & 56036 & 56036 & 0.00 & 74 & & 64613 & 64607 & 0.01 & 756 \\
& 0.6 & 63491 & 63485 & 0.01 & 98 & & 78754 & 77017 & 1.11 & 1161 \\
\hline 3 & 1 & 39902 & 39899 & 0.01 & 99 & 4 & 141923 & 141770 & 0.11 & 2733 \\
& 0.9 & 60629 & 60623 & 0.01 & 1239 & & 151974 & 151616 & 0.24 & 3397 \\
& 0.8 & 74191 & 73726 & 0.60 & 2829 & & 159565 & 158465 & 0.69 & 3511 \\
& 0.7 & 93683 & 90833 & 2.95 & 3540 & & 172430 & 166966 & 3.17 & 3600 \\
& 0.6 & 129088 & 112974 & 11.30 & 3564 & & 209493 & 184771 & 11.23 & 3600 \\
\hline 5 & 1 & 180552 & 179915 & 0.34 & 3431 & Mean & 80750 & 80591 & 0.09 & 1259 \\
& 0.9 & 191295 & 190204 & 0.56 & 3534 & & 94969 & 94678 & 0.16 & 1657 \\
& 0.8 & 197954 & 195221 & 1.35 & 3628 & & 104640 & 103774 & 0.54 & 2084 \\
& 0.7 & 213627 & 206606 & 3.25 & 3600 & & 120078 & 117010 & 1.88 & 2314 \\
& 0.6 & 266207 & 219855 & 16.04 & 3600 & & 149407 & 131621 & 7.94 & 2405 \\
\hline
\end{tabular}
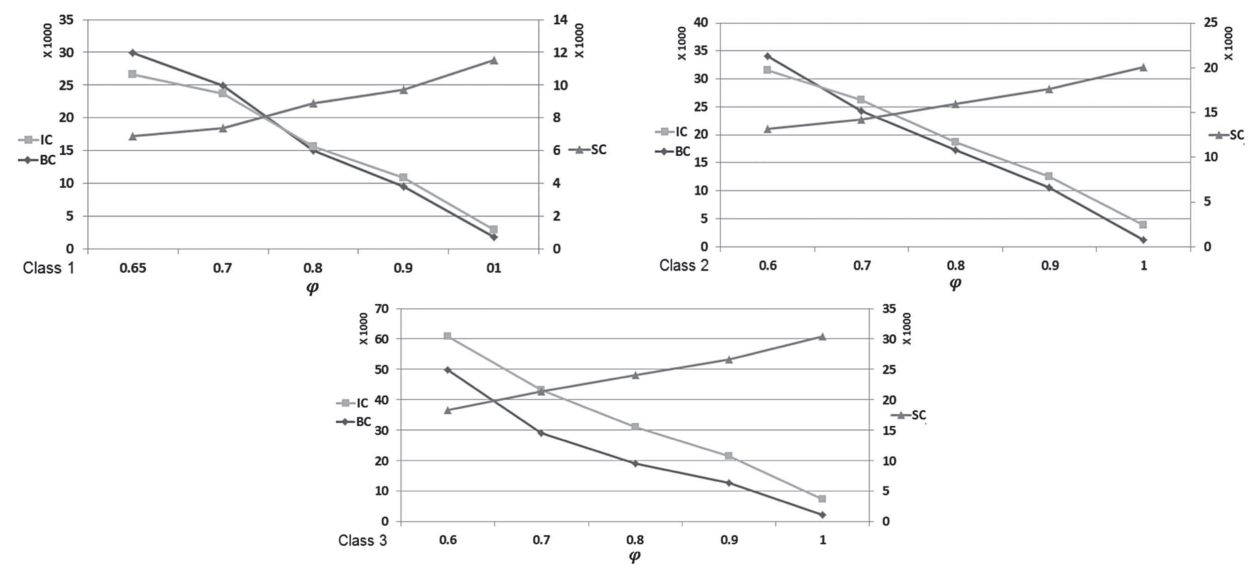

FIgURE 3. Inventory costs (IC), backlogging costs (BC) and setup costs (SC) obtained for some values of the parameter $\varphi$ for classes 1,2 and 3 .
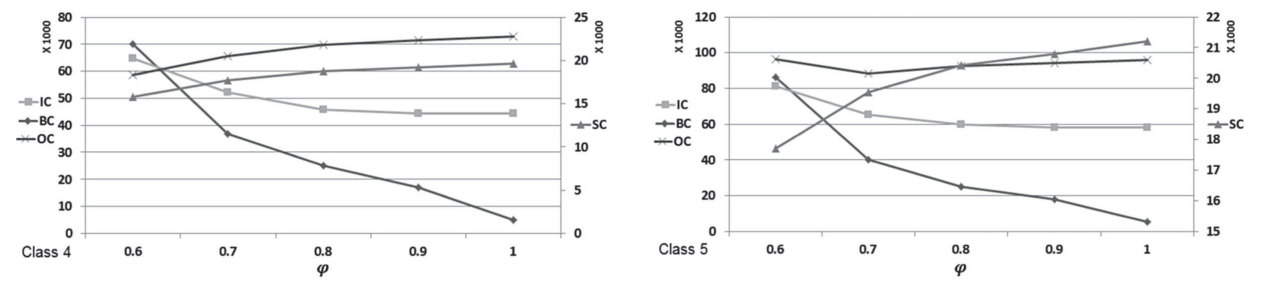

FiguRE 4. Inventory costs (IC), backlogging costs (BC), setup costs (SC), and costs to organize the lines (OC) obtained for some values of the parameter $\varphi$ for classes 3 and 4 . 


\section{Concluding Remarks}

In this paper, we studied a lot sizing and scheduling problem in which several production lines share the same scarce production resources. Due to the scarcity of resources, only a subset of the production lines can simultaneously operate in each period. We have combined techniques from the literature in order to propose nine mathematical optimization models to represent the problem. Moreover, we have presented a computational study carried out using a data set from the literature in order to identify the most promising formulations for the problem. Our computational study showed that an efficient model can be obtained from the CLSD model (introduced in [15]) by making explicit the binary production variables (as suggested in [23] for the single machine LSP), using the single commodity flow constraints to eliminate any sub-sequences (introduced in [11] and tested for the single machine LSP in [13]), and using the facility location reformulation [8], model FL$\mathrm{CLSD}_{\mathrm{SCF}}^{w}$. Moreover, we have presented a sensitivity analysis with respect to the parameter that controls the proportion of lines that can operate in each period, concluding that the problem becomes more difficulty to be solved when few production lines are available in each period.

There are a number of directions for potential future research. First of all, we note that heuristic procedures should be investigated with the aim of finding high quality solutions for large sized instances with more than 10 periods and 100 products. The computational performance of the FL-CLSD ${ }_{\mathrm{SCF}}^{w}$ encourages the proposition of MIP based heuristics. Moreover, we highlight that the nature of the problem with three main components: knapsack (resources capacity constraints), lot sizing, and scheduling; encourages the investigation of Lagrangian relaxation based approaches with the aim of obtaining subproblems in which these components could be separated.

Acknowledgements. This research was supported by the Universidade Federal de Mato Grosso do Sul and in part by the Brazilian agencies: National Council for Scientific and Technological Development-CNPq and São Paulo Research Foundation-FAPESP (grant 2016/01860-1). The research was conducted using the computational resources of the Center for Mathematical Sciences Applied to Industry - CeMEAI (FAPESP grant 2013/07375-0). The authors would like to thank the anonymous reviewers for their valuable insights, comments and suggestions.

\section{REFERENCES}

[1] M. Afzalirad and J. Rezaeian, Resource-constrained unrelated parallel machine scheduling problem with sequence dependent setup times, precedence constraints and machine eligibility restrictions. Comput. Ind. Eng. 98 (2016) 40-52.

[2] Z. Alipour, F. Jolai, E. Monabbati and N. Zaerpour, General lot-sizing and scheduling for perishable food products. RAIROOper. Res. 54 (2020) 913-931.

[3] B. Almada-Lobo, A. Clark, L. Guimarães, G. Figueira and P. Amorim, Industrial insights into lot sizing and scheduling modeling. Pesquisa Operacional 35 (2015) 439-46.

[4] C. Almeder and B. Almada-Lobo, Synchronisation of scarce resources for a parallel machine lotsizing problem. Int. J. Prod. Econ. 49 (2011) 7315-7335.

[5] A.R. Clark and S.J. Clark, Rolling-horizon lot-sizing when set-up times are sequence-dependent. Int. J. Prod. Econ. 38 (2000) $2287-2307$.

[6] K. Copil, M. Wörbelauer, H. Meyr and H. Tempelmeier, Simultaneous lotsizing and scheduling problems: a classification and review of models. OR Spec. 39 (2017) 1-64.

[7] E.D. Dolan and J.J. Moré, Benchmarking optimization software with performance profiles. Math. Program. 91 (2002) $201-213$.

[8] G.D. Eppen and R.K. Martin, Solving multi-item capacitated lot-sizing problems using variable redefinition. Oper. Res. 35 (1987) 832-848.

[9] B. Fleischmann and H. Meyr, The general lotsizing and scheduling problem. Oper. Res. Spek. 19 (1997) 11-21.

[10] H.Y. Fuchigami and S. Rangel, A survey of case studies in production scheduling: Analysis and perspectives. J. Comput. Sci. 25 (2018) 425-436.

[11] B. Gavish and S.C. Graves, The travelling salesman problem and related problems (1978).

[12] C.H. Glock, E.H. Grosse and J.M. Ries, The lot sizing problem: A tertiary study. Int. J. Prod. Econ. 155 (2014) $39-51$.

[13] L. Guimarães, D. Klabjan and B. Almada-Lobo, Modeling lotsizing and scheduling problems with sequence dependent setups. Eur. J. Oper. Res. 239 (2014) 644-662.

[14] M. Güngör, A.T. Ünal and Z.C. Taşkın, A parallel machine lot-sizing and scheduling problem with a secondary resource and cumulative demand. Int. J. Prod. Econ. 56 (2018) 3344-3357.

[15] K. Haase, Capacitated lot-sizing with sequence dependent setup costs. Oper. Res. Spek. 18 (1996) 51-59. 
[16] K. Haase and A. Kimms, Lot sizing and scheduling with sequence-dependent setup costs and times and efficient rescheduling opportunities. Int. J. Prod. Econ. 66 (2000) 159-169.

[17] R.J.W. James and B. Almada-Lobo, Single and parallel machine capacitated lotsizing and scheduling: New iterative mip-based neighborhood search heuristics. Comput. Oper. Res. 38 (2011) 1816-1825.

[18] G.M. Kopanos, L. Puigjaner and C.T. Maravelias, Production planning and scheduling of parallel continuous processes with product families. Ind. Eng. Chem. Res. 50 (2010) 1369-1378.

[19] E.L. Lawler, The traveling salesman problem: a guided tour of combinatorial optimization. Wiley-Interscience Series In Discrete Mathematics (1985).

[20] H. Meyr, Simultaneous lotsizing and scheduling by combining local search with dual reoptimization. Eur. J. Oper. Res. 120 (2000) 311-326.

[21] H. Meyr, Simultaneous lotsizing and scheduling on parallel machines. Eur. J. Oper. Res. 139 (2002) $277-292$.

[22] C.E. Miller, A.W. Tucker and R.A. Zemlin, Integer programming formulation of traveling salesman problems. JACM 7 (1960) 326-329.

[23] W.A. Oliveira and M.O. Santos, A new branching rule to solve the capacitated lot sizing and scheduling problem with sequence dependent setups. TEMA (São Carlos) 18 (2017) 515-529.

[24] W.A.O. Soler, M.O. Santos and K. Akartunali, Mip approaches for a lot sizing and scheduling problem on multiple production lines with scarce resources, temporary workstations, and perishable products. J. Oper. Res. Soc. (2019) 1-16.

[25] L.A. Wolsey, Mip modelling of changeovers in production planning and scheduling problems. Eur. J. Oper. Res. 99 (1997) $154-165$.

[26] J. Xiao, H. Yang, C. Zhang, L. Zheng and J.N.D. Gupta, A hybrid lagrangian-simulated annealing-based heuristic for the parallel-machine capacitated lot-sizing and scheduling problem with sequence-dependent setup times. Comput. Oper. Res. 63 (2015) $72-82$. 